Research Article

\title{
Permutation Invariant Strong Law of Large Numbers for Exchangeable Sequences
}

\author{
Stefan Tappe \\ Albert Ludwig University of Freiburg, Department of Mathematical Stochastics, Ernst-Zermelo-Strasse 1, \\ Freiburg D-79104, Germany \\ Correspondence should be addressed to Stefan Tappe; stefan.tappe@math.uni-freiburg.de
}

Received 12 July 2021; Accepted 5 November 2021; Published 27 November 2021

Academic Editor: Ramón M. Rodríguez-Dagnino

Copyright $(92021$ Stefan Tappe. This is an open access article distributed under the Creative Commons Attribution License, which permits unrestricted use, distribution, and reproduction in any medium, provided the original work is properly cited.

We provide a permutation invariant version of the strong law of large numbers for exchangeable sequences of random variables. The proof consists of a combination of the Komlós-Berkes theorem, the usual strong law of large numbers for exchangeable sequences, and de Finetti's theorem.

\section{Introduction}

Kolmogorov's strong law of large numbers (SLLN) for independent and identically distributed (i.i.d.) sequences of random variables has been generalized into several directions. It has, for example, been generalized for pairwise independent, identically distributed random variables in [1], for nonnegative random variables in [2], for dependent, mixing random variables in $[3,4]$, and for pairwise uncorrelated random variables in [5].

There is also a version of the SLLN for exchangeable sequences. More precisely, let $\left(\xi_{n}\right)_{n \in \mathbb{N}}$ be an exchangeable sequence of random variables on a probability space $(\Omega, F, \mathbb{P})$, let $\mathrm{E}$ be its exchangeable $\sigma$-algebra, and let T be its tail $\sigma$-algebra. If the sequence $\left(\xi_{n}\right)_{n \in \mathbb{N}}$ is integrable, then the SLLN for exchangeable sequences tells us that $\left(\xi_{n}\right)_{n \in \mathbb{N}}$ is almost surely Cesàro convergent; more precisely, we have the following result.

Proposition 1. Let $\left(\xi_{n}\right)_{n \in \mathbb{N}} \subset L^{1}$ be an exchangeable sequence of integrable random variables. Then, $\left(\xi_{n}\right)_{n \in \mathbb{N}}$ is $\mathbb{P}$-almost surely Cesàro convergent to the limit

$$
\xi=\mathbb{E}\left[\xi_{1} \mid \mathbb{E}\right]=\mathbb{E}\left[\xi_{1} \mid \mathrm{T}\right] .
$$

This result is well known; see, for example, [6], Example 12.15 , or [7], page 185. The goal of this note is to establish the following permutation invariant version of the SLLN for exchangeable sequences.

Theorem 1. Let $\left(\xi_{n}\right)_{n \in \mathbb{N}} \subset L^{1}$ be an exchangeable sequence of integrable random variables. We set $\xi:=\mathbb{E}\left[\xi_{1} \mid \mathrm{E}\right]$. Then, the following statements are true:

(1) For every subsequence $\left(n_{k}\right)_{k \in \mathbb{N}}$ and every permutation $\pi: \mathbb{N} \longrightarrow \mathbb{N}$, the sequence $\left(\xi_{n_{\pi(k)}}\right)_{k \in \mathbb{N}}$ is $\mathbb{P}$-almost surely Cesàro convergent to $\xi$

(2) For every permutation $\sigma: \mathbb{N} \longrightarrow \mathbb{N}$ and every subsequence $\left(m_{k}\right)_{k \in \mathbb{N}}$, the sequence $\left(\xi_{\sigma\left(m_{k}\right)}\right)_{k \in \mathbb{N}}$ is $\mathbb{P}$-almost surely Cesàro convergent to $\xi$

(3) We have $\mathbb{P}$-almost surely $\xi=\mathbb{E}\left[\xi_{n} \mid \mathrm{E}\right]=\mathbb{E}\left[\xi_{n} \mid \mathrm{T}\right]$ for each $n \in \mathbb{N}$

Intuitively, the statement of Theorem 1 is plausible. Indeed, de Finetti's theorem, which is stated as Theorem 3 in the following, provides a connection between exchangeable sequences and conditional i.i.d. sequences, and in the present situation, it implies that the sequence $\left(\xi_{n}\right)_{n \in \mathbb{N}}$ is i.i.d. given $\mathrm{E}$ or given $\mathrm{T}$.

Let us briefly indicate the main ideas for the proof of Theorem 1. Since exchangeability of the sequence is preserved under permutations, by Proposition 1, it follows that the sequences $\left(\xi_{n_{\pi(k)}}\right)_{k \in \mathbb{N}}$ and $\left(\xi_{\sigma\left(m_{k}\right)}\right)_{k \in \mathbb{N}}$ are almost surely 
Cesàro convergent. However, it is not clear whether the limits of these two sequences coincide with $\xi$ because their exchangeable $\sigma$-algebras can be different from E, and accordingly, their tail $\sigma$-algebras can be different from $\mathrm{T}$. Nevertheless, note that, by exchangeability of the sequence, all these limits have the same distribution.

In order to overcome the problem regarding the identification of the limits, we use the Komlós-Berkes theorem (see [8]), which is stated as Theorem 2 in the following. This result is an extension of Komlós's theorem (see [9]); see also [10], Thm. 5.2.1, for another extension of Komlós's theorem. The Komlós-Berkes theorem was also used in order to prove the von Weizsäcker theorem (see [11]); see also [10], Thm. 5.2.3, for a similar result and [12] for a note on the von Weizsäcker theorem.

Coming back to the identification of the limits, the Komlós-Berkes theorem provides us with a subsequence $\left(n_{k}\right)_{k \in \mathbb{N}}$ such that, for every permutation $\pi: \mathbb{N} \longrightarrow \mathbb{N}$, the sequence $\left(\xi_{n_{\pi(k)}}\right)_{k \in \mathbb{N}}$ is almost surely Cesàro convergent to the same limit. Using this result, in three steps, we will show that, for every subsequence and every permutation, the corresponding sequence is almost surely Cesàro convergent to the same limit and that this limit is given by $\xi$. For the identification of the limits, we use results about conditional expectations which are provided in the Appendix section.

\section{Proof of the Result}

Let $(\Omega, F, \mathbb{P})$ be a probability space. We denote by $L^{1}=$ $L^{1}(\Omega, F, \mathbb{P})$ the space of all equivalence classes of integrable random variables. Let $\left(\xi_{n}\right)_{n \in \mathbb{N}}$ be a sequence of random variables. Furthermore, let $\mathrm{E}$ be the exchangeable $\sigma$-algebra of the sequence $\left(\xi_{n}\right)_{n \in \mathbb{N}}$, and let T be the tail $\sigma$-algebra of the sequence $\left(\xi_{n}\right)_{n \in \mathbb{N}}$. We assume that the sequence $\left(\xi_{n}\right)_{n \in \mathbb{N}}$ is exchangeable; that is, for every finite permutation $\pi: \mathbb{N} \longrightarrow \mathbb{N}$, we have

$$
\mathbb{P} \circ\left(\left(\xi_{n}\right)_{n \in \mathbb{N}}\right)=\mathbb{P} \circ\left(\left(\xi_{\pi(n)}\right)_{n \in \mathbb{N}}\right),
$$

or equivalently, for all $k \in \mathbb{N}$, all pairwise different $n_{1}, \ldots, n_{k} \in \mathbb{N}$, and all pairwise different $m_{1}, \ldots, m_{k} \in \mathbb{N}$, we have

$$
\mathbb{P} \circ\left(\xi_{n_{1}}, \ldots, \xi_{n_{k}}\right)=\mathbb{P} \circ\left(\xi_{m_{1}}, \ldots, \xi_{m_{k}}\right)
$$

Remark 1. Note that, for every subsequence $\left(n_{k}\right)_{k \in \mathbb{N}}$ and every permutation $\pi: \mathbb{N} \longrightarrow \mathbb{N}$, the sequence $\left(\xi_{n_{\pi(k)}}\right)_{k \in \mathbb{N}}$ is also exchangeable. Accordingly, for every permutation $\sigma: \mathbb{N} \longrightarrow \mathbb{N}$ and every subsequence $\left(m_{k}\right)_{k \in \mathbb{N}}$, the sequence $\left(\xi_{\sigma\left(m_{k}\right)}\right)_{k \in \mathbb{N}}$ is also exchangeable.

\section{Lemma 1. The following statements are true:}

(1) For every subsequence $\left(n_{k}\right)_{k \in \mathbb{N}}$ and every permutation $\sigma: \mathbb{N} \longrightarrow \mathbb{N}$, there exist a permutation $\pi: \mathbb{N} \longrightarrow \mathbb{N}$ and a subsequence $\left(m_{k}\right)_{k \in \mathbb{N}}$ such that $\sigma\left(m_{k}\right)=n_{\pi(k)}$ for all $k \in \mathbb{N}$
(2) For every subsequence $\left(n_{k}\right)_{k \in \mathbb{N}}$ and every permutation $\pi: \mathbb{N} \longrightarrow \mathbb{N}$, there exists a permutation $\sigma: \mathbb{N} \longrightarrow \mathbb{N}$ such that $\sigma\left(n_{k}\right)=n_{\pi(k)}$ for all $k \in \mathbb{N}$

Proof

(1) We define the one-to-one map $\tau: \mathbb{N} \longrightarrow \mathbb{N}$ as

$$
\tau(k):=\sigma^{-1}\left(n_{k}\right), \quad \text { for each } k \in \mathbb{N} .
$$

Then, there exists a permutation $\pi: \mathbb{N} \longrightarrow \mathbb{N}$ such that $\tau(\pi(k))<\tau(\pi(k+1))$ for all $k \in \mathbb{N}$. Indeed, we define $\pi$ inductively as follows. Let $\pi(1) \in \mathbb{N}$ be the unique index such that

$$
\tau(\pi(1))=\min \{\tau(k): k \in \mathbb{N}\} .
$$

If $\pi(1), \ldots, \pi(p)$ are already defined for some $p \in \mathbb{N}$, then let $\pi(p+1) \in \mathbb{N}$ be the unique index such that

$\tau(\pi(p+1))=\min \{\tau(k): k \in \mathbb{N} \backslash\{\pi(1), \ldots, \pi(p)\}\}$.

Then, $\pi$ is a permutation. We define the subsequence $\left(m_{k}\right)_{k \in \mathbb{N}}$ as $m_{k}:=\tau(\pi(k))$ for each $k \in \mathbb{N}$. Then, we have $\sigma\left(m_{k}\right)=n_{\pi(k)}$ for each $k \in \mathbb{N}$.

(2) We define the permutation $\sigma: \mathbb{N} \longrightarrow \mathbb{N}$ as

$$
\sigma(m):= \begin{cases}n_{\pi(k)} & \text { if } m=n_{k} \text { for some } k \in \mathbb{N} \\ m, & \text { otherwise. }\end{cases}
$$

Then, we have $\sigma\left(n_{k}\right)=n_{\pi(k)}$ for all $k \in \mathbb{N}$.

For convenience of the reader, we state the Komlós-Berkes theorem and de Finetti's theorem before we provide the proof of Theorem 1.

Theorem 2. (Komlós-Berkes theorem). Let $\left(\xi_{n}\right)_{n \in \mathbb{N}} \subset L^{1}$ be a sequence of integrable random variables such that $\sup _{n \in \mathbb{N}} \mathbb{E}\left[\left|\xi_{n}\right|\right]<\infty$. Then, there exist a subsequence $\left(n_{k}\right)_{k \in \mathbb{N}}$ and an integrable random variable $\xi \in L^{1}$ such that, for every permutation $\pi: \mathbb{N} \longrightarrow \mathbb{N}$, the sequence $\left(\xi_{n_{\pi(k)}}\right)_{k \in \mathbb{N}}$ is $\mathbb{P}$-almost surely Cesàro convergent to $\xi$.

Proof. See [8].

Let $\mathrm{G} \subset \mathrm{F}$ be a sub- $\sigma$-algebra. A sequence $\left(\xi_{n}\right)_{n \in \mathbb{N}}$ of random variables is called independent and identically distributed (i.i.d.) given $\mathrm{G}$ if for every finite subset $I \subset \mathbb{N}$ and all Borel sets $B_{i} \in \mathrm{B}(\mathbb{R}), i \in I$, we have $\mathbb{P}$-almost surely

$\mathbb{P}\left(\bigcap_{i \in I}\left\{\xi_{i} \in B_{i}\right\} \mid \mathrm{G}\right)=\prod_{i \in I} \mathbb{P}\left(\xi_{i} \in B_{i} \mid \mathrm{G}\right)$ (independence given $\mathrm{G}$ ),

and for all $n, m \in \mathbb{N}$ and every Borel set $B \in \mathrm{B}(\mathbb{R})$, we have $\mathbb{P}$-almost surely 
$\mathbb{P}\left(\xi_{n} \in B \mid \mathrm{G}\right)=\mathbb{P}\left(\xi_{m} \in B \mid \mathrm{G}\right)($ identical distributions given $\mathrm{G})$.

Theorem 3 (de Finetti's theorem). Let $\left(\xi_{n}\right)_{n \in \mathbb{N}}$ be a sequence of random variables. Then, the following statements are equivalent:

(i) The sequence $\left(\xi_{n}\right)_{n \in \mathbb{N}}$ is exchangeable

(ii) There exists a sub- $\sigma$-algebra $\mathrm{G} \subset \mathrm{F}$ such that $\left(\xi_{n}\right)_{n \in \mathbb{N}}$ is i.i.d. given $\mathrm{G}$

If the previous conditions are fulfilled, then we can choose $\mathrm{G}=\mathrm{E}$ or $\mathrm{G}=\mathrm{T}$.

Proof. See, for example, Thm. 12.24 of [6].

Now, we are ready to provide the proof of Theorem 1.

Proof. of Theorem 1. By the Komlós-Berkes theorem (see Theorem 2), there exist a subsequence $\left(n_{k}\right)_{k \in \mathbb{N}}$ and an integrable random variable $\xi \in L^{1}$ such that, for every permutation $\pi: \mathbb{N} \longrightarrow \mathbb{N}$, the sequence $\left(\xi_{n_{\pi(k)}}\right)_{k \in \mathbb{N}}$ is $\mathbb{P}$-almost surely Cesàro convergent to $\xi$. Now, we proceed with the following three steps:

Step 1: first, we show that, for every permutation $\sigma: \mathbb{N} \longrightarrow \mathbb{N}$, the sequence $\left(\xi_{\sigma(n)}\right)_{n \in \mathbb{N}}$ is $\mathbb{P}$-almost surely Cesàro convergent to $\xi$. Indeed, by Lemma 1, there exist a permutation $\pi: \mathbb{N} \longrightarrow \mathbb{N}$ and a subsequence $\left(m_{k}\right)_{k \in \mathbb{N}}$ such that $\sigma\left(m_{k}\right)=n_{\pi(k)}$ for each $k \in \mathbb{N}$. By Remark 1 and Proposition 1, we have

$$
\xi=\mathbb{E}\left[\xi_{n_{\pi(1)}} \mid \mathrm{E}_{\left(n_{\pi(k)}\right)_{k \in \mathbb{N}}}\right]=\mathbb{E}\left[\xi_{n_{\pi(1)}} \mid \mathrm{T}_{\left(n_{\pi(k)}\right)_{k \in \mathbb{N}}}\right],
$$

where $\mathrm{E}_{\left(n_{\pi(k)}\right)_{k \in \mathbb{N}}}$ denotes the exchangeable $\sigma$-algebra of the sequence $\left(\xi_{n_{\pi(k)}}\right)_{k \in \mathbb{N}}$ and $\mathrm{T}_{\left(n_{\pi(k)}\right)_{k \in \mathbb{N}}}$ denotes the tail $\sigma$-algebra of the sequence $\left(\xi_{n_{(k)}}\right)_{k \in \mathbb{N}}$. Furthermore, by Remark 1 and Proposition 1, the sequence $\left(\xi_{\sigma(n)}\right)_{n \in \mathbb{N}}$ is $\mathbb{P}$-almost surely Cesàro convergent to the random variable

$$
\eta:=\mathbb{E}\left[\xi_{\sigma(1)} \mid \mathrm{E}_{(\sigma(n))_{n \in \mathbb{N}}}\right]=\mathbb{E}\left[\xi_{\sigma(1)} \mid \mathrm{T}_{(\sigma(n))_{n \in \mathbb{N}}}\right],
$$

where $\mathrm{E}_{(\sigma(n))_{n \in \mathbb{N}}}$ denotes the exchangeable $\sigma$-algebra of the sequence $\left(\xi_{\sigma(n)}\right)_{n \in \mathbb{N}}$ and $\mathrm{T}_{(\sigma(n))_{n \in \mathbb{N}}}$ denotes the tail $\sigma$-algebra of the sequence $\left(\xi_{\sigma(n)}\right)_{n \in \mathbb{N}}$. By de Finetti's theorem (see Theorem 3), we have

$$
\eta=\mathbb{E}\left[\xi_{n_{\pi(1)}} \mid \mathrm{E}_{(\sigma(n))_{n \in \mathbb{N}}}\right]=\mathbb{E}\left[\xi_{n_{\pi(1)}} \mid \mathrm{T}_{(\sigma(n))_{n \in \mathbb{N}}}\right] .
$$

Since $\mathrm{E}_{\left(n_{\pi(k)}\right)_{k \in \mathbb{N}}} \subset \mathrm{E}_{(\sigma(n))_{n \in \mathbb{N}}}$ and $\mathrm{T}_{\left(n_{\pi(k)}\right)_{k \in \mathbb{N}}} \subset \mathrm{T}_{(\sigma(n))_{n \in \mathbb{N}}}$ by (10), we obtain

$$
\xi=\mathbb{E}\left[\eta \mid \mathrm{E}_{\left(n_{\pi(k)}\right)_{k \in \mathbb{N}}}\right]=\mathbb{E}\left[\eta \mid \mathrm{T}_{\left(n_{\pi(k)}\right)_{k \in \mathbb{N}}}\right] .
$$

By exchangeability of the sequence $\left(\xi_{n}\right)_{n \in \mathbb{N}}$, we have
$\mathbb{P} \circ\left(\frac{1}{n} \sum_{i=1}^{n} \xi_{n_{\pi(i)}}\right)=\mathbb{P} \circ\left(\frac{1}{n} \sum_{i=1}^{n} \xi_{\sigma(i)}\right), \quad$ for each $n \in \mathbb{N}$,

and hence, by Proposition A.1, we obtain $\mathbb{P}$-almost surely $\xi=\eta$. In particular, if $\sigma=\mathrm{Id}$, then by (11) and de Finetti's theorem (see Theorem 3 ), we obtain $\mathbb{P}$-almost surely

$$
\xi=\eta=\mathbb{E}\left[\xi_{n} \mid \mathrm{E}\right]=\mathbb{E}\left[\xi_{n} \mid \mathrm{T}\right], \quad \text { for each } n \in \mathbb{N} .
$$

Step 2: now, let $\sigma: \mathbb{N} \longrightarrow \mathbb{N}$ be an arbitrary permutation, and let $\left(m_{k}\right)_{k \in \mathbb{N}}$ be an arbitrary subsequence. Then, the sequence $\left(\xi_{\sigma\left(m_{k}\right)}\right)_{k \in \mathbb{N}}$ is $\mathbb{P}$-almost surely Cesàro convergent to $\xi$. Indeed, by Step 1 and de Finetti's theorem (see Theorem 3), the sequence $\left(\xi_{\sigma(n)}\right)_{n \in \mathbb{N}}$ is $\mathbb{P}$-almost surely Cesàro convergent to

$$
\xi=\mathbb{E}\left[\xi_{\sigma(1)} \mid \mathrm{E}_{(\sigma(n))_{n \in \mathbb{N}}}\right]=\mathbb{E}\left[\xi_{\sigma\left(m_{1}\right)} \mid \mathrm{E}_{(\sigma(n))_{n \in \mathbb{N}}}\right] .
$$

Furthermore, by Remark 1 and Proposition 1, the sequence $\left(\xi_{\sigma\left(m_{k}\right)}\right)_{k \in \mathbb{N}}$ is $\mathbb{P}$-almost surely Cesàro convergent to the random variable

$$
\zeta:=\mathbb{E}\left[\xi_{\sigma\left(m_{1}\right)} \mid \mathrm{E}_{\left(\sigma\left(m_{k}\right)\right)_{k \in \mathbb{N}}}\right]
$$

Since $\mathrm{E}_{\left(\sigma\left(m_{k}\right)\right)_{k \in \mathbb{N}}} \subset \mathrm{E}_{(\sigma(n))_{n \in \mathbb{N}}}$, by (16), we obtain

$$
\zeta=\mathbb{E}\left[\xi \mid \mathrm{E}_{\left.\left(\sigma\left(m_{k}\right)\right)_{k \in \mathbb{N}}\right]} .\right.
$$

By exchangeability of the sequence $\left(\xi_{n}\right)_{n \in \mathbb{N}}$, we have

$$
\mathbb{P} \circ\left(\frac{1}{n} \sum_{i=1}^{n} \xi_{\sigma(i)}\right)=\mathbb{P} \circ\left(\frac{1}{n} \sum_{i=1}^{n} \xi_{\sigma\left(m_{i}\right)}\right), \quad \text { for each } n \in \mathbb{N}
$$

and hence, by Proposition A.1, we obtain $\mathbb{P}$-almost surely $\xi=\zeta$. Consequently, the sequence $\left(\xi_{\sigma\left(m_{k}\right)}\right)_{k \in \mathbb{N}}$ is $\mathbb{P}$-almost surely Cesàro convergent to $\xi$.

Step 3: now, let $\left(n_{k}\right)_{k \in \mathbb{N}}$ be an arbitrary subsequence, and let $\pi: \mathbb{N} \longrightarrow \mathbb{N}$ be an arbitrary permutation. By Lemma 1 , there exists a permutation $\sigma: \mathbb{N} \longrightarrow \mathbb{N}$ such that $\sigma\left(n_{k}\right)=n_{\pi(k)}$ for all $k \in \mathbb{N}$. Therefore, by Step 2, the sequence $\left(\xi_{n_{\pi(k}}\right)_{k \in \mathbb{N}}$ is $\mathbb{P}$-almost surely Cesàro convergent to $\xi$, which concludes the proof.

We can extend the statement of Theorem 1 as follows.

Proposition 2. Let $\left(\xi_{n}\right)_{n \in \mathbb{N}} \subset L^{1}$ be an exchangeable sequence of integrable random variables. We set $\xi:=\mathbb{E}\left[\xi_{1} \mid \mathrm{E}\right]$. Then, for every subsequence $\left(n_{k}\right)_{k \in \mathbb{N}}$ and all permutations $\pi, \sigma: \mathbb{N} \longrightarrow \mathbb{N}$, the sequence $\left(\xi_{\sigma\left(n_{\pi(k)}\right)}\right)_{k \in \mathbb{N}}$ is $\mathbb{P}$-almost surely Cesàro convergent to $\xi$. Furthermore, we have $\mathbb{P}$-almost surely $\xi=\mathbb{E}\left[\xi_{n} \mid \mathrm{E}\right]=\mathbb{E}\left[\xi_{n} \mid \mathrm{T}\right]$ for each $n \in \mathbb{N}$. 
Proof. By Lemma 1, there exists a permutation $\tau: \mathbb{N} \longrightarrow \mathbb{N}$ such that $\tau\left(n_{k}\right)=n_{\pi(k)}$ for all $k \in \mathbb{N}$. The mapping $\rho: \mathbb{N} \longrightarrow \mathbb{N}$ given by $\rho:=\sigma \circ \tau$ is also a permutation, and we have $\sigma\left(n_{\pi(k)}\right)=\rho\left(n_{k}\right)$ for all $k \in \mathbb{N}$. Therefore, applying Theorem 1 concludes the proof.

We conclude this section with the following consequence regarding Komlós's theorem for exchangeable sequences, namely, let $\left(\xi_{n}\right)_{n \in \mathbb{N}} \subset L^{1}$ be an exchangeable sequence of random variables. Then, Theorem 1 shows that both extensions of Komlós's theorem (the Komlós-Berkes theorem from [8], which we have stated as Theorem 2, and Thm. 5.2.1 of [10]) are true with the original sequence $\left(\xi_{n}\right)_{n \in \mathbb{N}}$; that is, we do not have to pass to a subsequence $\left(\xi_{n_{k}}\right)_{k \in \mathbb{N}}$.

\section{Appendix}

\section{Results about Conditional Expectations}

We require the following results about conditional expectations. Since these results were not immediately available in the literature, we provide the proofs. For the following, let $\mathrm{G} \subset \mathrm{F}$ be a sub- $\sigma$-algebra.

Lemma A.1. Let $X \in L^{2}$ be a square-integrable random variable such that $\mathbb{P} \circ X=\mathbb{P} \circ \mathbb{E}[X \mid \mathrm{G}]$. Then, we have P-almost surely $X=\mathbb{E}[X \mid \mathrm{G}]$.

Proof. Setting $Y:=\mathbb{E}[X \mid \mathrm{G}]$, we have $\mathbb{E}\left[X^{2}\right]=\mathbb{E}\left[Y^{2}\right]$, and hence,

$$
\begin{aligned}
\mathbb{E}\left[(X-Y)^{2}\right]= & \mathbb{E}\left[X^{2}\right]-2 \mathbb{E}[X Y]+\mathbb{E}\left[Y^{2}\right]=2 \mathbb{E}\left[X^{2}\right] \\
& -2 \mathbb{E}[\mathbb{E}[X Y \mid \mathrm{G}]] \\
= & 2 \mathbb{E}\left[X^{2}\right]-2 \mathbb{E}[Y \mathbb{E}[X \mid \mathrm{G}]]=2 \mathbb{E}\left[X^{2}\right] \\
& -2 \mathbb{E}\left[Y^{2}\right]=0,
\end{aligned}
$$

completing the proof.

Lemma A.2. Let $X \in L^{1}$ be a nonnegative random variable, and let $\varphi: \mathbb{R}^{+} \longrightarrow \mathbb{R}^{+}$be a concave function such that $\mathbb{P}$-almost surely

$$
\mathbb{E}[\varphi(X)]=\mathbb{E}[\varphi(\mathbb{E}[X \mid G])] .
$$

Then, we have $\mathbb{P}$-almost surely

$$
\mathbb{E}[\varphi(X) \mid \mathrm{G}]=\varphi(\mathbb{E}[X \mid \mathrm{G}]) .
$$

Proof. By Jensen's inequality for concave functions and conditional expectations, we have $\mathbb{P}$-almost surely

$$
\mathbb{E}[\varphi(X) \mid \mathrm{G}] \leq \varphi(\mathbb{E}[X \mid \mathrm{G}]) .
$$

Suppose that (A.3) does not hold true. Then, we have $\mathbb{P}$-almost surely

$$
\varphi(\mathbb{E}[X \mid \mathrm{G}])-\mathbb{E}[\varphi(X) \mid \mathrm{G}] \in L_{+}^{0} \backslash\{0\},
$$

where $L_{+}^{0}$ denotes the convex cone of all equivalence classes of nonnegative random variables. Hence, we obtain $\mathbb{P}$-almost surely

$$
\mathbb{E}[\varphi(X)]=\mathbb{E}[\mathbb{E}[\varphi(X) \mid G]]<\mathbb{E}[\varphi(\mathbb{E}[X \mid G])],
$$
which contradicts (A.2).

Lemma A.3. Let $X \in L^{1}$ be an integrable random variable such that

$$
\mathbb{P} \circ X=\mathbb{P} \circ \mathbb{E}[X \mid G]
$$

Then, we have $\mathbb{P}$-almost surely $X=\mathbb{E}[X \mid \mathrm{G}]$.

Proof. First, we assume that $X \in L^{1}$ is nonnegative. Let $n \in \mathbb{N}$ be arbitrary. By (A.7) and Lemma A.2, we have $\mathbb{P}$-almost surely

$$
\mathbb{E}[X \mid \mathrm{G}] \wedge n=\mathbb{E}[X \wedge n \mid \mathrm{G}] .
$$

Therefore, by taking into account (A.7), we have

$$
\mathbb{P} \circ(X \wedge n)=\mathbb{P} \circ(\mathbb{E}[X \mid G] \wedge n)=\mathbb{P} \circ \mathbb{E}[X \wedge n \mid G] .
$$

Since $X \wedge n \in L^{2}$, by Lemma A.1 and (A.8), we deduce that $\mathbb{P}$-almost surely

$$
X \wedge n=\mathbb{E}[X \wedge n \mid \mathrm{G}]=\mathbb{E}[X \mid \mathrm{G}] \wedge n .
$$

Since $n \in \mathbb{N}$ was arbitrary, it follows that $\mathbb{P}$-almost surely $X=\mathbb{E}[X \mid G]$.

Now, let $X \in L^{1}$ be arbitrary. Since

$$
\begin{aligned}
& \mathbb{E}[X \mid \mathrm{G}]^{+}=\mathbb{E}\left[X^{+} \mid \mathrm{G}\right], \\
& \mathbb{E}[X \mid \mathrm{G}]^{-}=\mathbb{E}\left[X^{-} \mid \mathrm{G}\right],
\end{aligned}
$$

by (A.7), we have

$$
\begin{aligned}
& \mathbb{P} \circ X^{+}=\mathbb{P} \circ \mathbb{E}\left[X^{+} \mid \mathrm{G}\right], \\
& \mathbb{P} \circ X^{-}=\mathbb{P} \circ \mathbb{E}\left[X^{-} \mid \mathrm{G}\right] .
\end{aligned}
$$

By the first part of the proof, we deduce that $\mathbb{P}$-almost surely $X^{+}=\mathbb{E}\left[X^{+} \mid \mathrm{G}\right]$ and $X^{-}=\mathbb{E}\left[X^{+} \mid \mathrm{G}\right]$, and hence, $X=\mathbb{E}[X \mid \mathrm{G}]$.

Proposition A.1. Let $\left(X_{n}\right)_{n \in \mathbb{N}}$ and $\left(Y_{n}\right)_{n \in \mathbb{N}}$ be sequences of random variables, and let $X \in L^{1}$ be an integrable random variable. We assume that $\mathbb{P} \circ X_{n}=\mathbb{P} \circ Y_{n}$ for each $n \in \mathbb{N}$ and that $X_{n} \longrightarrow{ }^{\text {a.s. }} X$ and $Y_{n} \longrightarrow{ }^{\text {a.s. }} \mathbb{E}[X \mid \mathrm{G}]$ as $n \longrightarrow \infty$. Then, we have $\mathbb{P}$-almost surely $X=\mathbb{E}[X \mid \mathrm{G}]$.

Proof. Noting that $\mathbb{P} \circ X=\mathbb{P} \circ \mathbb{E}[X \mid G]$, this is a consequence of Lemma A.3.

\section{Data Availability}

No data were used to support this study.

\section{Conflicts of Interest}

The author declares that there are no conflicts of interest. 


\section{Acknowledgments}

The author gratefully acknowledges the financial support from the Deutsche Forschungsgemeinschaft (DFG, German Research Foundation) (Project no. 444121509).

\section{References}

[1] N. Etemadi, "An elementary proof of the strong law of large numbers," Zeitschrift für Wahrscheinlichkeitstheorie und Verwandte Gebiete, vol. 55, no. 1, pp. 119-122, 1981.

[2] N. Etemadi, "On the laws of large numbers for nonnegative random variables," Journal of Multivariate Analysis, vol. 13, no. 1, pp. 187-193, 1983.

[3] A. Kuczmaszewska, "The strong law of large numbers for dependent random variables," Statistics \& Probability Letters, vol. 73, no. 3, pp. 305-314, 2005.

[4] A. Kuczmaszewska, "On the strong law of large numbers for $\phi$-mixing and $\rho$-mixing random variables," Acta Mathematica Hungarica, vol. 132, no. 1-2, pp. 174-189, 2011.

[5] M. Janisch, "Kolmogorov's strong law of large numbers holds for pairwise uncorrelated random variables," Theory of Probability \& Its Applications, vol. 66, no. 2, pp. 263-275, 2021.

[6] A. Klenke, Probability Theory. A Comprehensive Course, Springer, London, UK, 2nd edition, 2014.

[7] J. F. C. Kingman, "Uses of exchangeability," Annals of Probability, vol. 6, no. 2, pp. 183-197, 1978.

[8] I. Berkes, "An extension of the Komlós subsequence theorem," Acta Mathematica Hungarica, vol. 55, no. 1-2, pp. 103-110, 1990.

[9] J. Komlós, “A generalization of a problem of Steinhaus," Acta Mathematica Hungarica, vol. 18, no. 1-2, pp. 217-229, 1967.

[10] Y. M. Kabanov and M. Safarian, Markets with Transaction Costs. Mathematical Theory, Springer, Berlin, Germany, 2009.

[11] H. v. Weizsäcker, "Can one drop $\mathrm{L}^{1}$-boundedness in Komlós's subsequence theorem?" The American Mathematical Monthly, vol. 111, no. 10, pp. 900-903, 2004.

[12] S. Tappe, "A note on the von Weizsäcker theorem," Statistics \& Probability Letters, vol. 168, p. 6, Article ID 108926, 2021. 